\title{
A Multi-Dimensional Analysis of Reliability Criteria: From Deterministic N-1 to a Probabilistic Approach
}

\author{
Evelyn Heylen, Marten Ovaere*, Stef Proost*, Geert Deconinck, Dirk Van Hertem \\ KU Leuven, Department of Electrical Engineering, EnergyVille \\ Kasteelpark Arenberg 10 PB 2445, 3001 Leuven-Heverlee \\ ${ }^{*} K U$ Leuven, Department of Economics \\ Naamsestraat 69, 3000 Leuven
}

\begin{abstract}
This paper proposes a classification of reliability criteria for power systems based on four characteristics: (i) the set of considered system states, (ii) the objective function, (iii) the allowed real-time actions and (iv) optional non-technical constraints. Because selecting a reliability criterion involves a trade-off between multiple opposing objectives, this paper suggests the use of five performance indicators to evaluate the performance of reliability criteria: (i) expected total cost, (ii) service reliability level, (iii) inequality between consumers in terms of service reliability, (iv) availability, integrity and confidentiality of input information and (v) ease-of-use. Case studies for a five-node and 24-node test system illustrate the multi-dimensional analysis of six reliability criteria. These criteria range from the deterministic N-1 criterion to an unconstrained probabilistic criterion that aims to minimize expected total cost. The analysis finds that moving from deterministic to probabilistic reliability criteria decreases expected total cost. The largest savings of expected total cost are due to a trade-off between preventive and corrective actions. A smaller portion of savings is due to the inclusion of load curtailment actions in the trade-off on top of preventive and corrective control. Limits on individual or aggregate service unreliability levels decrease service unreliability, but increase expected total costs when compared to an unconstrained probabilistic approach.

Keywords: Power system operation, power system reliability, reliability management, N-1 reliability criterion, probabilistic reliability criteria.
\end{abstract}

\footnotetext{
* Corresponding author

Email address: evelyn.heylen@esat.kuleuven.be (Evelyn Heylen)
} 


\section{Introduction}

Reliability management based on the N-1 criterion has been frequently questioned during the last decade. Studies argue that a radically different approach may be required to integrate renewable energy sources and smart grid technologies in a cost-effective way [1, 2, 3, 4, 5]. They argue that probabilistic

5 reliability criteria are better suited to meet the current challenges of the electricity transmission system: uncertain and variable demand and supply, decentralized decision makers, highly interconnected networks, difficulties in building new lines and a general trend towards a more efficient use of the transmission system [6].

A large number of papers has been studying probabilistic reliability criteria as alternatives to constrained optimal power flow [24]. They mainly focus on the theoretical part of the problem, i.e., how to obtain a more detailed simulation of decision-making behavior.

Although significant scientific work has already been done on probabilistic reliability management, directly moving from a deterministic N-1 criterion to a probabilistic approach in short-term reliability System Operators (TSOs) ${ }_{1}^{1}$ seldom use probabilities in their short-term reliability management, except for some TSOs that exclude very rare events from the contingency list or treat extreme weather events differently due to higher associated failure probabilities [9, 25. NERC focuses on the development of

\footnotetext{
${ }^{1}$ We use the term 'TSO' throughout this paper, but the core of our analysis does not change with the level of ownership unbundling of the network (full ownership unbundling, independent system operator or independent transmission operator) or with the geographical area of focus (e.g. in North America: independent system operator or regional transmission organization)
} 
probabilistic adequacy indicators, but their security indicators are still deterministic in nature [26]. in reliability calculations for power system planning and development, e.g., to determine the generation reserve in the system development phase or to evaluate network reinforcements [27, 28. TSOs are not eager to change their reliability management and criterion, amongst others due to the satisfactory results obtained in the past and the ease-of-use of the N-1 reliability criterion. Probabilistic reliability criteria, in contrast, are perceived to be more complex.

To facilitate the paradigm shift in reliability management, this paper assesses characteristics of reliability criteria to bridge the gap between the deterministic N-1 approach and an unconstrained probabilistic approach that aims at minimizing total system cost without imposing additional constraints on service reliability This paper makes two contributions to the literature. First, this paper distinguishes reliability criteria between the N-1 approach and an unconstrained probabilistic approach by proposing a classification of reliability criteria based on four characteristics: (i) the set of considered system states, (ii) the objective function, (iii) the allowed real-time actions and (iv) optional non-technical constraints. This opens up a range of reliability criteria that can be studied by TSOs, academics and regulators and that can serve as intermediate steps towards using probabilistic criteria in practice. Second, to convince decision makers to consider alternatives for the deterministic N-1 criterion, this paper proposes an assessment of different reliability criteria along five dimensions: (i) expected total cost, (ii) service unreliability, (iii) inequality between consumers in terms of service reliability, (iv) availability, integrity and confidentiality of input information and (v) ease-of-use. Case studies in a five-node and 24-node test system illustrate the multi-dimensional performance assessment of six proposed reliability criteria. The design of the simulation model used in the case study is based on the conceptual framework proposed in [31]. The case study does not intend to identify the fundamentally optimal reliability criterion for actual large-scale systems, but intends to illustrate the classification framework and multi-dimensional analysis and indicate general characteristics and performance of different reliability criteria. The proposed analysis enables a better understanding of the different reliability criteria proposed in the literature and the difficulties for these criteria to be

\footnotetext{
${ }^{2}$ From an economic perspective, the optimal reliability criterion minimizes total system cost. Minimization of total system cost can be considered as an approximation for social surplus maximization under certain assumptions [29].

${ }^{3}$ Service reliability is defined as the ability to adequately satisfy the demand under given operating conditions for a given time interval. Power system reliability on the contrary is the ability of an electric power system to perform a required function under given conditions for a given time interval [30. The required function and the given conditions are specified by the applied reliability criterion.
} 
adequately adopted by TSOs that continue to use the N-1 criterion. TSOs and regulators can use the methods proposed in this paper to carry out a multi-dimensional analysis for their own systems.

Section 2 presents the classification of reliability criteria and describes six reliability criteria that range from the deterministic N-1 reliability criterion to an unconstrained probabilistic reliability criterion for short-term reliability management. Section 3 discusses the five indicators to evaluate the performance of reliability criteria. The six reliability criteria are evaluated along the five indicators in a case study in Section 4 . Section 5 discusses the results, while Section 6 concludes.

\section{Reliability criteria}

Reliability criteria guide reliability management of TSOs, from long-term system development to short-term operational planning and real-time operation [6]. In each of these planning horizons, the TSO continuously takes actions to minimize the cost of satisfying the reliability criterion. This paper focuses on the minimization problem of the TSO in operational planning and real-time operation.

$$
\begin{aligned}
& \min _{\boldsymbol{a}_{\boldsymbol{p}}, \boldsymbol{a}_{\boldsymbol{c}}^{\boldsymbol{s}}, \boldsymbol{P}_{\boldsymbol{c}}^{\boldsymbol{s}}}\left[C\left(\boldsymbol{a}_{\boldsymbol{p}}, \boldsymbol{a}_{\boldsymbol{c}}^{\boldsymbol{s}}, \boldsymbol{P}_{\boldsymbol{c}}^{\boldsymbol{s}}\right)\right] \\
& \quad \text { s.t. } G_{s}\left(\boldsymbol{a}_{\boldsymbol{p}}, \boldsymbol{a}_{\boldsymbol{c}}^{\boldsymbol{s}}, \boldsymbol{P}_{\boldsymbol{c}}^{\boldsymbol{s}}\right)=0, H_{s}\left(\boldsymbol{a}_{\boldsymbol{p}}, \boldsymbol{a}_{\boldsymbol{c}}^{\boldsymbol{s}}, \boldsymbol{P}_{\boldsymbol{c}}^{\boldsymbol{s}}\right) \geq 0 \quad \forall s \in S
\end{aligned}
$$

In operational planning and real-time operation, the TSO's objective is to minimize total cost limits (inequality constraints $H_{s}$ ) [32. During operational planning, the TSO takes the most costeffective preventive actions $\boldsymbol{a}_{\boldsymbol{p}}$ to ensure that these constraints are met in all considered system states $s \in S 4^{4}$ The set of considered system states depends on the reliability criterion.

If contingencies happen in real time and preventive actions turn out to be insufficient, the TSO can take corrective actions $\boldsymbol{a}_{\boldsymbol{c}}^{\boldsymbol{s}}$ or resort to load curtailment $\boldsymbol{P}_{\boldsymbol{c}}^{\boldsymbol{s}} \mathrm{W}^{5}$ They choose the cheapest actions that are within the constraints of the applied reliability criterion to make sure operational limits are still met. Unconsidered system states could lead to uncontrolled brownouts or blackouts, if corrective actions are not able to deal with the realized real-time system state.

The remainder of this section formulates and discusses six reliability criteria 6 Each reliability crite-

\footnotetext{
${ }^{4}$ Examples of available actions in the operational planning stage are generation redispatch, branch switching, phase shifting transformer tap changing, and ensuring the availability of upward and downward reserves 33 .

${ }^{5}$ Possible corrective actions are branch switching, secondary voltage control, capacitor and reactor bank switching, the use of upward and downward reserves, phase shifting transformer tap changing and load curtailment 32 .

${ }^{6}$ To simplify the notation, $G_{s}()$ represents both the equality and inequality constraints in system state $s$.
} 
rion consists of a cost minimization subject to constraints that represent the power flow equations and operational limits. The reliability criteria are defined by four characteristics: (i) the set of considered system states $S$, (ii) the allowed TSO actions in considered system states, (iii) the objective function to minimize and (iv) optional non-technical constraints. Table 1 summarizes the analyzed reliability criteria along these four characteristics 7 The reliability criteria are formulated mathematically as optimization programs for reasons of clarity and comparability. Nevertheless, the classification framework is not restricted to reliability criteria that are cast mathematically as optimization problems, due to the link between reliability assessment and optimal power flow formulations [2].

\section{1. $N$-1 reliability criterion}

The N-1 criterion states that after an unexpected outage of a single system component, the transmission system should still be able to operate within limits without load curtailment. A direct link between the preventive and corrective stage is not made if not required and the system is secured ahead of real time, if possible. The TSO's objective function is deterministic and limited to minimizing the cost of preventive actions. The expected costs of corrective and curtailment actions in real time are not explicitly considered. The set of considered system states consists of all N-1 contingencies and is usually called the N-1 contingency set. In case of N-1 network contingencies $S_{N-1, \text { network }}$, the network should be able to accommodate all resulting flows. The mathematical formulation of the N-1 reliability criterion is:

$$
\begin{aligned}
\min _{\boldsymbol{a}_{\boldsymbol{p}}} & {\left[C_{\text {prev }}\left(\boldsymbol{a}_{\boldsymbol{p}}\right)\right] } \\
& \text { s.t. } G_{s_{n}}\left(\boldsymbol{a}_{\boldsymbol{p}}\right)=0 \quad \forall s_{n} \in S_{N-1, \text { network }}
\end{aligned}
$$

In case of N-1 generation contingencies $S_{N-1, \text { generation, }}$, real-time corrective actions $\boldsymbol{a}_{\boldsymbol{c}}^{\boldsymbol{s}}$, like upward and downward use of reserves, are needed to restore the balance between demand and supply.

$$
G_{s}\left(\boldsymbol{a}_{\boldsymbol{p}}, \boldsymbol{a}_{\boldsymbol{c}}^{\boldsymbol{s}}\right)=0 \quad \forall s \in S_{N-1, \text { generation }}
$$

In any case, load curtailment is not allowed in N-1 system states (i.e. in both network and generation N-1 contingencies). The N-1 reliability criterion does not explicitly prepare for multiple contingencies.

\footnotetext{
${ }^{7}$ The discussed reliability criteria mainly focus on risk-neutral reliability management. Alternatively, risk-averse objective functions can be considered that are typically implemented using robust optimization techniques [34].
} 
In these cases, load curtailment could turn out to be required to prevent a blackout in real time operation. Nowadays, the N-1 criterion is the most widely used security criterion in operational planning 35 .

\subsection{Deterministic reliability criterion with a different set of considered system states}

The mathematical formulation of this reliability criterion is similar to that of the N-1 reliability criterion, the primary difference being that the set of considered system states $S$ is allowed to differ from the N-1 contingency set.

$$
\begin{aligned}
\min _{\boldsymbol{a}_{\boldsymbol{p}}} & {\left[C_{\text {prev }}\left(\boldsymbol{a}_{\boldsymbol{p}}\right)\right] } \\
& \text { s.t. } G_{s}\left(\boldsymbol{a}_{\boldsymbol{p}}\right)=0 \quad \forall s \in S_{p} \\
& \text { s.t. } G_{s}\left(\boldsymbol{a}_{\boldsymbol{p}}, \boldsymbol{a}_{\boldsymbol{c}}^{\boldsymbol{s}}\right)=0 \quad \forall s \in S \backslash S_{p}
\end{aligned}
$$

This reliability criterion requires a TSO to minimize its cost of preventive actions $C_{\text {prev }}$ while meeting the constraints for a subset of considered system states $S_{p}$ with preventive actions only and for the remaining considered system states $S \backslash S_{p}$ with both preventive and corrective actions to restore the balance between demand and supply, e.g., if generator outages occur. The set of considered system states $S$ could be anything. For example, include N-1 network contingencies, but exclude generator and busbar failures from the N-1 contingency set [25, p.25]; increase the contingency set to include multiple dependent failures with a high probability of occurrence; change the contingency set over time (e.g. including double-circuit failures only during adverse weather) or between regions (e.g. including more contingencies for urban areas or business districts); etc. In addition to contingencies, the set of considered system states could also include deviations from the expected operating condition, like forecast errors of demand and intermittent supply. In its most general form, the set of considered system states $S$ is the Cartesian product of credible contingencies and considered operating conditions. $S$ is always a subset of the infinite set of all possible future real-time states and contingencies (RT). Fig. 1 illustrates different sets of considered system states in the Cartesian plane of contingencies and operating conditions: the set of $\mathrm{N}-1$ network contingencies $S_{N-1, \text { network }}$, the set of N-1 contingencies $S_{N-1}$, a set of contingencies $S_{c}$, and a general set $S$ of contingencies and operating conditions.

It is also possible that (well-informed) state selection leads to a set of considered system states in which not all of the N-1 contingencies are included. This might be, for instance, because the probability of occurrence or the impact of the excluded states is too low. The impact on the performance indicators 


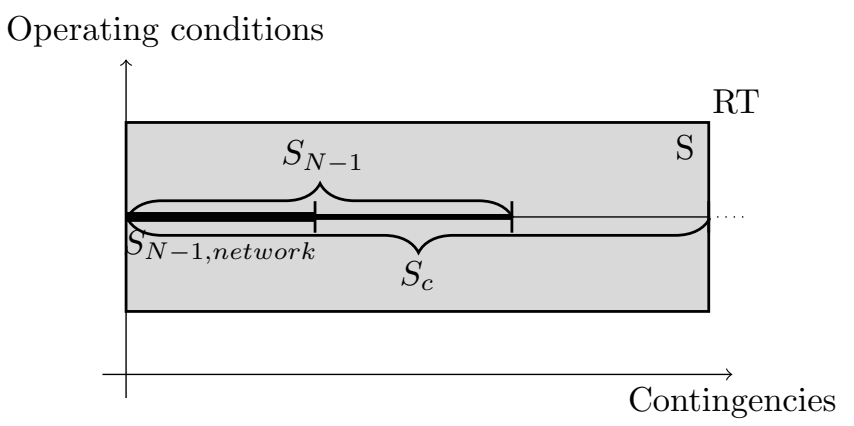

Figure 1: Different sets of considered system states in the Cartesian plane of contingencies and operating conditions. $S$ refers to the grey area, whereas $R T$ refers to the space of states.

of such sets is hard to predict, as this is an interaction between the impact of the additional and removed operating states.

These alternative deterministic reliability criteria are already in use nowadays. The N-2 criterion is used in parts of the United Kingdom where a higher security is required. It prescribes that the system should be secured against the simultaneous loss of two independent system components during normal operation [36]. In parts of Norway, for instance in very remote regions, the N-0 criterion is applied. This might result in a partial interruption of the power supply to consumers due to the loss of a single element causing violation of operational limits. Some TSOs do not have the strict interpretation that no loss of load is accepted, but have a certain limit to the loss of load that is accepted in the case of a failure [25. Some TSOs apply the N-1-1 criterion or consecutive N-2 criterion, which considers a sequence of events consisting of the initial loss of a single generator or transmission component, followed by system adjustments, followed by another loss of a single generator or transmission component [37. The TPL-001-1 standard of NERC specifies a deterministic criterion based on four categories of events with different post-fault requirements [38, 39].

\subsection{Probabilistic reliability criterion without load curtailment in considered states $S$}

If the TSO takes the expected cost of corrective actions in considered system states $s \in S$ into account in operational planning, its objective function becomes probabilistic. The TSO simulates which actions it will take in each of the considered system states and the expected cost is calculated as the product of the cost of actions in each state and its associated probability of occurrence. First, suppose that load curtailment is not allowed in considered system states. The mathematical formulation 
becomes:

$$
\begin{gathered}
\min _{\boldsymbol{a}_{\boldsymbol{p}}, \boldsymbol{a}_{\boldsymbol{c}}^{\boldsymbol{s}}}\left[C_{\text {prev }}\left(\boldsymbol{a}_{\boldsymbol{p}}\right)+\sum_{s \in S} \pi_{s}\left(C_{\text {cor }}\left(\boldsymbol{a}_{\boldsymbol{c}}^{\boldsymbol{s}}\right)\right)\right] \\
\text { s.t. } G_{s}\left(\boldsymbol{a}_{\boldsymbol{p}}, \boldsymbol{a}_{\boldsymbol{c}}^{\boldsymbol{s}}\right)=0 \quad \forall s \in S
\end{gathered}
$$

Where $\pi_{s}$ is the probability of system state $s$ and $C_{c o r}\left(\boldsymbol{a}_{\boldsymbol{c}}^{\boldsymbol{s}}\right)$ is the cost of corrective actions in state $s$.

The difference between this criterion and the previous criterion is that a TSO now incorporates the effect of its preventive actions on the cost of its corrective actions, instead of just checking if the constraints are met. This allows an explicit trade-off between preventive and corrective actions, as first introduced by Monticelli et al. [40].

\subsection{Unconstrained probabilistic reliability criterion}

If, in addition, load curtailment is allowed in considered system states and the TSO also takes the expected cost of load curtailment into account in its operational planning minimization, this results in an unconstrained probabilistic reliability criterion. The mathematical formulation is:

$$
\begin{gathered}
\min _{\boldsymbol{a}_{\boldsymbol{p}}, \boldsymbol{a}_{\boldsymbol{c}}^{\boldsymbol{s}}, \boldsymbol{P}_{\boldsymbol{c}}^{\boldsymbol{s}}}\left[C_{\text {prev }}\left(\boldsymbol{a}_{\boldsymbol{p}}\right)+\sum_{s \in S} \pi_{s}\left(C_{\text {cor }}\left(\boldsymbol{a}_{\boldsymbol{c}}^{\boldsymbol{s}}\right)+C_{\text {curt }}\left(\boldsymbol{P}_{\boldsymbol{c}}^{\boldsymbol{s}}\right)\right)\right] \\
\text { s.t. } G_{s}\left(\boldsymbol{a}_{\boldsymbol{p}}, \boldsymbol{a}_{\boldsymbol{c}}^{\boldsymbol{s}}, \boldsymbol{P}_{\boldsymbol{c}}^{\boldsymbol{s}}\right)=0 \quad \forall s \in S
\end{gathered}
$$

Where $\boldsymbol{P}_{\boldsymbol{c}}^{\boldsymbol{s}}$ represents the load curtailment [MW] of consumers in state $s$. The difference between this criterion and the previous criterion is that the effect of preventive actions on the cost of corrective actions and load curtailment is incorporated. This allows an explicit trade-off between preventive, corrective and curtailment actions. Strbac et al. introduced the trade-off between preventive, corrective and curtailment actions in 4. Condren et al. 41] and He et al. 18, elaborated on the complete description of the reliability criterion in a security constrained optimal power flow formululation.

\subsection{Probabilistic reliability criterion with a constraint on the aggregate service reliability level}

While the unconstrained probabilistic reliability criterion aims at minimizing the expected total cost (ETC), it can also reduce the service reliability level considerably [42]. Therefore, social and political concerns could lead to the addition of a constraint on the value of the aggregate service reliability level $\bar{P}$. Such a constraint limits the decrease of the service reliability level. If the constraint 
is binding, ETC will be higher. The mathematical formulation becomes:

$$
\begin{aligned}
\min _{\boldsymbol{a}_{\boldsymbol{p}}, \boldsymbol{a}_{\boldsymbol{c}}^{\boldsymbol{s}}, \boldsymbol{P}_{\boldsymbol{c}}^{\boldsymbol{s}}} & {\left[C_{\text {prev }}\left(\boldsymbol{a}_{\boldsymbol{p}}\right)+\sum_{s \in S} \pi_{s}\left(C_{\text {cor }}\left(\boldsymbol{a}_{\boldsymbol{c}}^{\boldsymbol{s}}\right)+C_{\text {curt }}\left(\boldsymbol{P}_{\boldsymbol{c}}^{\boldsymbol{s}}\right)\right)\right] } \\
\text { s.t. } & G_{s}\left(\boldsymbol{a}_{\boldsymbol{p}}, \boldsymbol{a}_{\boldsymbol{c}}^{\boldsymbol{s}}, \boldsymbol{P}_{\boldsymbol{c}}^{\boldsymbol{s}}\right)=0 \quad \forall s \in S \\
\text { s.t. } & \sum_{j \in J} \sum_{s \in S} \pi_{s} P_{c}^{s}(j) \leq \bar{P}
\end{aligned}
$$

Where $J$ represents the set of all consumers.

Although the non-technical constraints in this paper are expressed in terms of a particular reliability indicator, i.e., expected power not supplied, other reliability indicators, such as Loss of Load Probability

\footnotetext{
${ }^{8}$ On top of these constrained individual reliability levels, a constraint on the aggregate reliability level is included in the reliability management approach introduced in 43 .
}

Capitanescu introduced the use of constraints on individual reliability levels in [43]. 8 
Table 1: Summary of reliability criteria.

\begin{tabular}{l|cccccc}
\hline & \multicolumn{7}{|c}{ Criteria } & & \\
& $(\mathrm{a})$ & $(\mathrm{b})$ & $(\mathrm{c})$ & $(\mathrm{d})$ & $(\mathrm{e})$ & $(\mathrm{f})$ \\
\hline 1. Set of considered states & $S_{N-1}$ & $S_{c}$ & $S$ & $S$ & $S$ & $S$ \\
2. Curtailment allowed in $S$ & no & no & no & yes & yes & yes \\
3. Objective function & Det. $^{1}$ & Det. & Prob. $^{2}$ & Prob. & Prob. & Prob. \\
4. Non-technical constraints & $/$ & $/$ & $/$ & $/$ & $\bar{P}$ & $\bar{P}_{c}(j)$ \\
\hline
\end{tabular}

1 Deterministic 2 Probabilistic
(a) Deterministic with N-1 contingency set
(b) Deterministic with different set of considered states
(c) Probabilistic without curtailment
(d) Unconstrained probabilistic
(e) Probabilistic with aggregated constraint
(f) Probabilistic with individual constraint

\section{Performance evaluation of reliability criteria}

The performance of power system reliability criteria is multi-faceted and several opposing objectives need to be considered. Some of them can be quantified, while others are qualitative in nature. The multi-dimensional performance assessment proposed in this paper is based on five indicators with different units. Decision makers need to make a trade-off between the different dimensions when moving towards probabilistic approaches.

\subsection{Quantitative indicators}

There are three important quantitative indicators that determine the performance of different reliability criteria: expected total cost $(E T C)$, relative load curtailment $(R L C)$ and inequality between consumers in terms of service unreliability $\left(U_{E N S}\right)$.

Socio-economic performance is evaluated in terms of expected total cost. It is the sum of the cost of preventive actions, the cost of corrective actions and interruption costs. Interruption costs equal the amount of load curtailed times the value of lost load (VOLL) and represent the consequences of an interruption for the consumers $9^{9}$ The total cost $(T C)$ at a certain time $t$ and real-time state $r t$ is equal to:

$$
T C(t, r t)=C_{p r e v}\left(\boldsymbol{a}_{\boldsymbol{p}}(t, r t)\right)+C_{c o r}\left(\boldsymbol{a}_{\boldsymbol{c}}(t, r t)\right)+\sum_{j \in J} V O L L(j, t) \cdot P_{c}(j, t, r t)
$$

where $J$ is the set of consumers in the system. The expected total cost $(E T C)$ over all times $t$ and

\footnotetext{
${ }^{9}$ VOLL depends on many factors, such as interruption and consumer characteristics [42]. In this paper, VOLL only depends on consumer type and interruption time.
} 
real-time states $r t$ is equal to:

$$
E T C=\sum_{t \in T} \pi(t) \sum_{r t \in R T} \pi(r t \mid t) \cdot T C(t, r t)
$$

Where $\pi(t)$ is the probability of being at time $t$ and $\pi(r t \mid t)$ the probability of being in real-time state $r t$ at time $t$. The set $T$ contains for example all 8760 hours of a typical year or a representative subset of time instants. Since the set $R T$ is the infinite set of all possible contingencies and all possible operating conditions, 20 is in practice evaluated for a finite subset $R T^{\prime} \subseteq R T$, where the set of considered system states $S \subseteq R T^{\prime}$.

The service reliability in the system is here expressed in terms of relative load curtailment. Relative load curtailment $R L C$ is rescaled to the equivalent number of minutes curtailed in a year:

$$
R L C=\left(\frac{P_{\text {curt }}}{P_{D}}\right) \cdot 8760 \cdot 60 \quad[\mathrm{~min} / \text { year }]
$$

Where $P_{D}$ is the total demand and $P_{\text {curt }}$ the curtailed load.

Reliability criteria that result in large differences of load curtailment between consumers or between nodes might not be socially acceptable. Therefore, equality is another important aspect to consider in the selection of a practical reliability criterion. Inequality can be quantified in a single number by using a Gini-based performance index $U_{E N S}$. The inequality index $U_{E N S}$ is defined as [44]:

$$
U_{E N S}=\left|1-\sum_{k}\left(D_{k}-D_{k-1}\right) \cdot\left(E_{k}+E_{k-1}\right)\right|
$$

Where $D$ is the cumulative share of demand, $E$ the cumulative share of energy not supplied (for example over a year) and $k$ an index counting over consumers or consumer groups. The groups are ordered based on decreasing reliability values. If $U_{E N S}$ equals 0 , unreliability is distributed equally among the entities under study, i.e. nodes, consumer groups or individual consumers ${ }^{10}$ If the inequality index $U_{E N S}$ is closer to 1, all interruptions are concentrated in one or a few consumer groups or nodes [44.

\footnotetext{
${ }^{10}$ Note that the inequality index can not be calculated if reliability is $100 \%$ for all consumer groups in all nodes. In that case inequality $U_{E N S}$ equals 0 .
} 


\subsection{Qualitative indicators}

Besides three quantitative performance indicators, two qualitative indicators should be considered: (i) the availability, integrity and confidentiality of the required input information and (ii) ease-of-use. processes require advanced computations to ensure the practicability of the criteria.

\footnotetext{
${ }^{11}$ We have reduced the subjectivity in the assessment of the ease-of-use to the minimum by considering three objective parameters, i.e., whether the formulation is deterministic or probabilistic in nature, the number of considered system states and the amount of information to take into account in the decision making. To establish the credibility of these results in a practical context, a transparent, objective and verifiable process is required.
} 


\section{Case study}

This section applies the proposed performance evaluation to the six reliability criteria introduced

Table 3: Generation data

\begin{tabular}{cccc}
\hline Node & $\begin{array}{c}\text { Capacity } \\
{[\mathrm{MW}]}\end{array}$ & Type & $\begin{array}{c}\text { Outage } \\
\text { probab. }{ }^{1}\end{array}$ \\
\hline 1 & 40 & conventional & $6.2 \mathrm{E}-7$ \\
1 & 40 & conventional & $6.2 \mathrm{E}-7$ \\
1 & 10 & conventional & $6.2 \mathrm{E}-3$ \\
1 & 20 & wind & $6.2 \mathrm{E}-3$ \\
2 & 40 & conventional & $6.2 \mathrm{E}-7$ \\
2 & 20 & conventional & $6.2 \mathrm{E}-3$ \\
2 & 20 & wind & $6.2 \mathrm{E}-3$ \\
2 & 20 & wind & $6.2 \mathrm{E}-3$ \\
2 & 20 & wind & $6.2 \mathrm{E}-3$ \\
2 & 5 & conventional & $6.2 \mathrm{E}-3$ \\
2 & 5 & conventional & $6.2 \mathrm{E}-3$ \\
\hline \multicolumn{5}{c}{ Contingencies are assumed to be indepen- } \\
dent. & Outage probabilities are calculated \\
based on failure rates and repair times \\
estimated based on the data in [45].
\end{tabular}

Total system demand is based on the hourly load profile defined in [46. For simplification a year is represented by a set of 72 time instants $T$, each with their probability of occurrence ${ }^{12}$ Total system demand at each of the 72 time instants is calculated as the mean over all valid hours. By weighing the outcomes of the different time instants by their yearly frequency of occurrence, the quantitative indicators are evaluated for a year. The simulation is repeated for a more stressed and a less stressed case, as defined in Table 4

\footnotetext{
${ }^{12}$ The 72 time instants represent 6 periods in the year (winter, early spring, late spring, summer, early autumn and late autumn), 3 types of days (weekday, Saturday and Sunday) and 4 times during the day (night, morning, noon and evening). Independent one hour time steps are considered in the simulation. The impact of the interruption duration on the value of lost load is thus not explicitly considered. Further details regarding the test system can be found in [47.
} 
Table 4: Summary of the three cases for the sensitivity analysis

\begin{tabular}{l|ccc}
\hline & More stressed & Base case & Less stressed \\
\hline Load & $105 \%$ & $100 \%$ & $95 \%$ \\
Failure rates & $150 \%$ & $100 \%$ & $75 \%$ \\
Repair times & $150 \%$ & $100 \%$ & $75 \%$ \\
Line rating & $91 \%$ & $100 \%$ & $136 \%$ \\
\hline
\end{tabular}

This numerical illustration uses VOLL data from Norway [48. Two consumer types are considered: residential and non-residential consumers. The share of residential and non-residential demand in total system demand changes throughout the year, as presented in [42].

In this paper, an analytical non-sequential state enumeration technique is applied [47. Analytical state enumeration enables the assessment of the relative performance of reliability criteria for a characteristic set of system states. The computation time is reasonable due to the parallelization options of the non-sequential evaluation technique, which does not consider correlations between time instants. The quantitative performance indicators are evaluated for a set of time instants $T$ for which forecast values of load and renewable power generation are given. Corrective reliability management is simulated for a set $R T^{\prime}$ of real time realizations for each time instant in the set $T$. This set is the Cartesian product of possible contingencies and real time operating states ${ }^{13}$ The latter are conditional upon the forecast values of net demand.

Preventive and corrective reliability management are modeled using a DC security constrained optimal power flow in AMPL 49, taking into account the specifics per criterion as discussed in Section 4.2 Available actions are generation redispatch, branch switching, phase shifting transformer tap changing and load curtailment depending on the applied criterion [50. The simulations are done in Matlab using an interface with AMPL and CPLEX as solver.

\subsection{Implementation of the reliability criteria}

Criterion (a) in Table 1 considers all N-1 branch and generator outages in the preventive decision stage. All these operating states are considered to be equally probable. Load curtailment is avoided for this contingency set and all consumers are treated equally. The objective is to secure the system

\footnotetext{
${ }^{13}$ Most probable contingencies up to cumulative probability of $99.73 \%$ and 11 realizations of net total demand based on a normal distribution of which the mean equals the forecast value and the coefficient of variation is $4 \%$. This set of system states is larger than the set $\mathrm{S}$ considered in decision making to evaluate reliability management also in system states that are not considered in advance. The number of realizations is not optimized. The realizations are symmetrically and equidistantly chosen around the forecast value, with a maximal deviation of $+/-3 \sigma$ [47].
} 
preventively as much as possible and corrective actions are considered as a last resort. The above also holds for criterion (b), but the set of considered contingencies is different.

Criteria (b) - (f) consider the most probable contingencies up to a cumulative probability of $99.7 \%$.

This contingency set does not contain the entire N-1 contingency set in the case study, as the failure of some large conventional generator units is not considered due to their low probability of failure.

In addition to the contingency set, reliability criteria (c) - (f) also consider forecast errors of load ${ }^{14}$ Their set of considered system states $S$ is the Cartesian product of the elements of the contingency set and 7 possible real time realizations of net total demand. These realizations are determined based on a normal distribution with the forecast value of net total demand as mean and a coefficient of variation of $4 \%$. Probabilistic criteria (c) - (f) take into account exact probabilities.

While probabilistic criterion (c) tries to avoid load curtailment, criteria (d) - (f) allow load curtailment if this tends to be more cost effective. However, criteria (e) - (f) have upper limits on the amount of load curtailment, i.e. on aggregated load curtailment for criterion (e) and individual limits for criterion (f). In the latter case, the limit on aggregated load curtailment is distributed over the consumer groups according to their demand share 15

The set of operating states used in criterion (b), $S_{c}$, and criteria (c) - (f), $S$, is graphically illustrated in Fig. 2. Some system states included in the N-1 contingency set $S_{N-1}$ are not included in the alternative sets $S_{c}$ and $S$, due to their low probability of occurrence

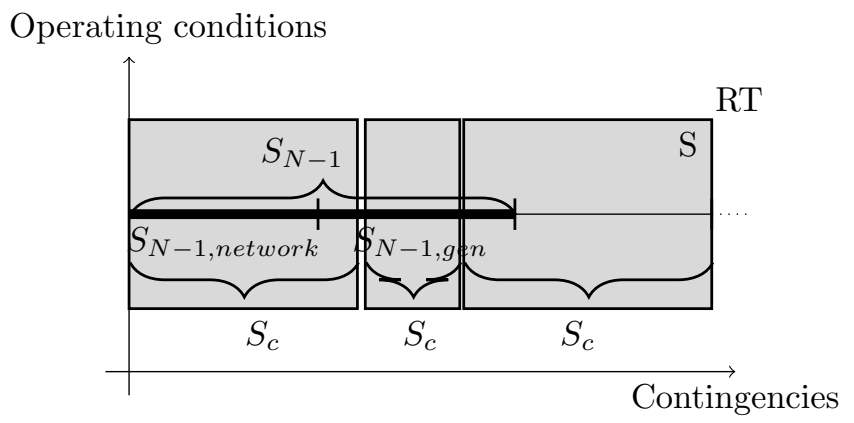

Figure 2: Graphical representation of the set of operating states used in criterion (a) $S_{N-1}$, criterion (b) $S_{c}$ and criteria (c) - (f) $S$. $S$ refers to the grey area, whereas $R T$ refers to the space of states.

\footnotetext{
${ }^{14}$ In the case study, the set $S_{c}$ of criterion (b) only considers contingencies and does not take preventive actions to prepare for forecast errors.

${ }^{15}$ The limit on aggregated load curtailment equals $0.4 \mathrm{MW}$ in this case study.
} 


\subsection{Results}

Table 5 summarizes the performance of the six reliability criteria in the base case of the 5 -node system, as indicated in Table 4

The first row of Table 5 shows that expected total cost decreases when moving from criterion (a) to criterion (d) and increases again when imposing restrictions on the aggregate (crit. (e)) and individual (crit. (f)) service reliability level. Criterion (b) results in a lower ETC than criterion (a), because in our case study the set of considered system states $S$ excludes low-probability contingencies that require costly preventive actions. Generally, the change of ETC from (a) to (b) depends on the current performance of the $\mathrm{N}-1$ criterion. If the $\mathrm{N}-1$ criterion is too stringent, enlarging the set of considered system states leads to even higher total costs. If the N-1 criterion is too loose, enlarging the set of considered system states could lead to lower total costs. Next, ETC decreases by moving from deterministic criteria (a) and (b) to probabilistic criterion (c), because more information is included in the operational planning decision. Criterion (c) makes an explicit trade-off between preventive and expected corrective actions. By additionally allowing load curtailment, if this is less costly than alternative corrective actions, criterion (d) leads to even lower ETC. In this case study, most of the decreased ETC is due to a better trade-off between preventive and expected corrective actions (crit. (c)). A smaller part is due to additionally allowing load curtailment in considered system states on top of preventive and corrective control (crit (d)). The higher VOLL is compared to the costs of other corrective actions, the larger this effect [42. The ETC of criteria (e) and (f) can be anywhere between the ETC of criteria (c) and (d). The more stringent the imposed reliability constraint, the higher the ETC. Individual constraints on the service reliability level (crit. (f)) always lead to a higher ETC than an aggregate constraint (crit. (e)), as load curtailment of low-VOLL consumers is now substituted for corrective or preventive actions, or load curtailment of consumers with a higher VOLL. To summarize:

$$
E T C_{d} \leq E T C_{e} \leq E T C_{f} \leq E T C_{c} \leq E T C_{b} \text { and } E T C_{a}
$$

The second row of Table 5 shows the unreliability of the electricity service for each of the six criteria. The effect of a particular reliability criterion on the reliability level is closely related to its effect on ETC. Criterion (b) has a slightly higher unreliability than criterion (a), because in our case study not all elements in the N-1 contingency set $S_{N-1}$ are part of the set $S$, as shown in Fig. 3. Moving from deterministic to probabilistic criteria has a mixed effect on service reliability, depending on the exact formulation of the criterion. Criterion (c) leads to an equal or lower unreliability than criterion 
(b), as in the latter the considered contingencies are a subset of the set of considered system states in the former. Even though for criteria (a) - (c) curtailment is not allowed in considered system states, unreliability is not zero because load curtailment could still be necessary in non-considered states. If load curtailment is allowed in considered states (crit. (d)), ETC decreases, but at the expense of a highly increased unreliability. Constraints on the reliability level (crit. (e) and (f)) decrease the service unreliability, but at the expense of a higher ETC. To summarize:

$$
R L C_{c} \leq R L C_{d} \geq R L C_{e} \geq R L C_{f}
$$

$U_{E N S}$ in Tables 5,6 and 7 shows the inequality between consumers in terms of service reliability. Inequality between consumers depends on the relative amount of load curtailment, but also on the system design and system strength. The results of the more stressed case in Table 6 indicate that deterministic criteria lead to higher ETC and lower inequality, while probabilistic criteria that allow load curtailment lead to lower ETC and higher inequality. The reason is that deterministic criteria treat all consumers equally, while these probabilistic criteria differentiate between consumers or nodes in terms of VOLL. Inequality is lower if individual constraints on load curtailment are applied (crit. f) than for criteria (d) and (e), but at the expense of higher ETC. This is the case, because the inequality resulting from the differentiation in VOLL is reduced by these individual limits, which are based on consumers' demand share. If systems are less stressed, such as in Table 5 and the less stressed case in Table 6. RLC is lower for criteria (a) - (c) and possibly concentrated in a reduced number of consumers. This increases the inequality between consumers resulting from criteria (a) - (c) and reduces the difference with criteria (d) - (f).

The fourth row of Table 5 shows that issues related to the availability, integrity and confidentiality of input information increase from left to right. Probabilistic approaches (c) - (f) require more input information than deterministic approaches. Firstly, exact probabilities of failures and cost information should be known. Moreover, forecast errors can be considered and for criteria (e) and (f) appropriate limits on load curtailment should be determined. Part of this input information is currently not available for TSOs and might be hard to obtain in practice. Failure probabilities might be imprecise, as well as forecast errors, which might lead to inappropriate reliability management.

The last rows of Table 5 show that the ease of using the considered criteria decreases from left to right, because more operating states need to be considered, more information needs to be taken into account and the probabilistic nature of criteria (c) - (f) adds a layer of complexity to reliability 
management.

Table 5: Performance evaluation of the six considered reliability criteria in the base case of the five-node test system: The first three indicators give a numerical value and the last two indicators are expressed as a value $(-/+/++/+++)$ from worst to best (relative).

\begin{tabular}{|c|c|c|c|c|c|c|}
\hline \multirow{2}{*}{ Base case } & \multicolumn{6}{|c|}{ Criteria of Table 1} \\
\hline & (a) & (b) & (c) & $(d)$ & (e) & (f) \\
\hline 1. $E T C[\%]$ & 100 & 87.34 & 34.62 & 26.63 & 33.83 & 34.50 \\
\hline 2. $R L C[\mathrm{~min}]$ & 0.0046 & 0.0077 & 0.0046 & 18.87 & 1.83 & 0.19 \\
\hline 3. $U_{E N S}[/]$ & 0.741 & 0.6128 & 0.569 & 0.811 & 0.794 & 0.604 \\
\hline $\begin{array}{l}\text { 4. Input information } \\
\text { needs and availability }\end{array}$ & +++ & ++ & + & + & - & - \\
\hline 5. Ease-of-use & +++ & ++ & + & + & - & - \\
\hline 5a. Type & Det. & Det. & Prob. & Prob. & Prob. & Prob. \\
\hline 5b. \# of states ${ }^{1}$ & 19 & 28 & 196 & 196 & 196 & 196 \\
\hline 5c. \# info & +++ & ++ & + & + & - & - \\
\hline
\end{tabular}

${ }^{1}$ Dependent on the state selection algorithm and corresponding to the sets $S_{N-1}, S_{c}$ and $S$ for resp. criteria (a), (b) and (c) - (f) containing a combination of pre-fault states and considered contingencies.

2 The numerical values cannot be generalized to real systems, but the trends between reliability criteria can.

Table 6 summarizes the results of the sensitivity analysis for the more stressed and less stressed cases defined in Table 4. In the more stressed case, the relative difference in total system cost between other criteria is distributed over a larger set of consumers.

Strbac et al. [1] questioned the fairness of deterministic criteria. Our analysis adds to this point that unconstrained probabilistic criteria can reduce inequality in less stressed cases at the cost of higher RLC, as illustrated in the 24-node RTS. In stressed conditions, however, probabilistic criteria lead to 
higher inequality than deterministic approaches, as illustrated in the five-node test system. In those cases, the level of inequality can be reduced using well-chosen individual service reliability limits.

Table 6: Sensitivity of the six considered reliability criteria in a more stressed case and a less stressed case of the five-node test system

\begin{tabular}{l|cccccc}
\hline $\begin{array}{l}\text { More stressed } \\
\text { case }\end{array}$ & (a) & (b) & (c) & (d) & (e) & (f) \\
\hline$E T C[\%]$ & 100 & 84.34 & 37.93 & 27.89 & 37.04 & 37.42 \\
$R L C[\mathrm{~min}]$ & 0.0796 & 0.0862 & 0.0632 & 20.3 & 1.92 & 1 \\
$U_{E N S}[/]$ & 0.353 & 0.473 & 0.3569 & 0.815 & 0.693 & 0.581 \\
\hline Less stressed & \multicolumn{7}{|c}{ Criteria of Table 1 } \\
case & (a) & (b) & (c) & $(\mathrm{d})$ & $(\mathrm{e})$ & $(\mathrm{f})$ \\
\hline$E T C[\%]$ & 100 & 88.36 & 32.42 & 25.00 & 31.66 & 32.33 \\
$R L C[\mathrm{~min}]$ & 0.0002 & 0.0016 & 0.0001 & 17.7 & 1.82 & 0.189 \\
$U_{E N S}[/]$ & 0.718 & 0.476 & 0.694 & 0.814 & 0.829 & 0.629 \\
\hline
\end{tabular}

Table 7: Performance evaluation of the six considered reliability criteria in IEEE 24 node RTS system.

\begin{tabular}{l|cccccc}
\hline & \multicolumn{6}{|c}{ Criteria of Table 1} \\
& (a) & (b) & (c) & (d) & (e) & (f) \\
\hline$E T C[\%]$ & 100 & 93.00 & 88.72 & 81.41 & 84.38 & 88.38 \\
$R L C[\mathrm{~min}]$ & 0.291 & 0.291 & 0.291 & 14.12 & 9.35 & 0.313 \\
$U_{E N S}[/]$ & 0.967 & 0.966 & 0.967 & 0.883 & 0.922 & 0.963 \\
\hline
\end{tabular}

\section{Discussion}

Despite the advantage of probabilistic criteria to decrease the cost of reliability management, the $\mathrm{N}-1$ criterion, or a variation of this, is still widely used by network operators in short-term system operation. Barriers against implementing probabilistic approaches are mainly due to the lack of available and accurate input information, transparency issues and complexity of the decision making. Advanced computations can support the complex decision making in probabilistic approaches involving optimal trade-offs, but concessions in modeling accuracy required to obtain a practical computational complexity hamper the application in real-life systems. Nevertheless, the intermediate steps proposed in this paper can help to gradually move towards probabilistic approaches. The Icelandic transmission system operator has illustrated how the transition towards probabilistic reliability criteria can be initiated by applying probabilistic reliability management based on probabilistic security assessment combined with operator experience in the control room. To further improve the reliability criteria, the 
quantitative and qualitative aspects in the proposed multi-dimensional performance assessment should be weight against each other.

\subsection{Barriers against implementing probabilistic reliability management approaches and criteria}

The main barriers for probabilistic criteria are the lack of available and accurate input information, as well as the difficulty to understand and use the approach. These are the factors summarized by the two qualitative indicators. To advance towards probabilistic reliability criteria, both the input information and the ease-of-use should be improved.

Data to obtain adequate input information can be gathered at a decreasing cost due to advances in communication and information technologies. Devices to measure climatic data, real-time voltage and current data, and regional demand and generation data are being installed in many countries. In an initial stage, aggregate information can be used, such as location-independent forecast errors of load and renewable generation, a time-independent VOLL, and constant failure probabilities of lines and generation. More detailed information can be included in the decision-making process once it is available, such as spatially-dependent forecast errors, time-dependent VOLL , and failure probabilities that depend on component lifetime and external conditions. However, the accuracy of this information influences the decision-making behavior. Therefore, it is important to check the sensitivity of the performance of reliability criteria with respect to the exactness of the provided information. If for instance both the sensitivity of the results to the accuracy of the failure probabilities and the uncertainty on the accuracy of the failure probabilities is high, accounting for imprecise probability in the decision making might be favorable [51].

Another barrier for probabilistic criteria is their alleged lack of transparency. Comparing transparency of probabilistic and deterministic approaches is not straightforward, because they are both transparent about different aspects. Deterministic criteria are transparent about when and why reliability actions are undertaken and about which contingencies do not lead to interruptions. However, they are less transparent about the risk level and the incentive used in contingency selection. Transparency of probabilistic criteria depends on their practical implementation. Probabilistic criteria can be transparent about objectives, constraints, trade-offs or the risk level while making a trade-off between different aspects such as service reliability, equality and efficiency. However, unconstrained probabilistic criteria are in general less transparent about the set of contingencies that is preventively secured and the selected reliability actions are much more dependent on the system state.

Probabilistic criteria are also inherently more complex than their deterministic counterparts, as 
probabilistic approaches make decisions based on trade-offs instead of on a binary criterion. They have a steep learning curve and the ease-of-use is low nowadays due to the lack of practical experience. However, the transition from deterministic to probabilistic approaches can be facilitated by taking intermediate steps between the currently used N-1 criterion and an unconstrained probabilistic criterion. The amount of input information and complexity can be gradually increased, in line with experience gained. A first step is to define an adequate set of system states to consider. This set can be probability based or can be a time-dependent set of considered contingencies or system states based on a trade-off between the cost of preventive and corrective actions ${ }^{16}$ This requires appropriate state selection techniques [52. This approach retains the simplicity of deterministic criteria, while employing the cost-reducing trade-offs of probabilistic criteria. A well selected set of considered system states can for instance reduce conservatism regarding low probability N-1 states, which may be costly to secure. In a next step, expected interruption costs can be added to this trade-off. In later steps, explicit probabilistic trade-offs can be introduced in practical short-term reliability management. The optimal trade-offs in probabilistic approaches, however, increase the complexity, which asks for advanced computations to support the decision making of the system operator in practice.

\subsection{Computational complexity of reliability management approaches}

Although the formulation in terms of optimization formulations helps to achieve a concise manuscript, solving an optimization problem is not necessary to apply all reliability criteria discussed in this paper in practice. The deterministic N-1 criterion (crit. (a)) is currently applied in practice based on contingency analysis and operator experience. The contingency analysis has parallelization opportunities, which reduces the computation time. An optimization is not strictly required due to the binary nature of the criterion, i.e., the system is either reliable if all prescribed conditions are satisfied or not reliable otherwise. Criterion (b), which only differs with criterion (a) regarding the considered set of system states, neither needs a SCOPF to be applied in practice.

Probabilistic criteria, on the contrary, involve optimal trade-offs between preventive, corrective and curtailment actions that increase the complexity of decision making. To optimize the trade-offs while satisfying operational limits and reliability constraints in the considered system states, system operators benefit from the support of a SCOPF. The computational challenges of the SCOPF in a reallife context are however significant. First, the problem is large-scale due to the nature of the stochastic

\footnotetext{
${ }^{16}$ For example, a different set for high and low demand, or normal and adverse weather.
} 
optimization model. Second, an AC implementation is required, involving nonconvex constraints due to the power flow physics. Third, the SCOPF formulation involves a large number of binary variables to model amongst others topological actions, PST tap settings, start-up and shut-down of generation and binary risk and reliability constraints. This results in a large-scale, non-convex mixed integer problem.

The original (non-convex) AC power flow formulation can be solved quickly to local optimality or slowly to global optimality, whereas convex approximations can be solved in polynomial time to global optimality with guaranteed convergence [53]. Literature has focussed on finding strong convex semidefinite and second-order cone approximations for non-convex problems [54]. These convex approximations are tractable, but in general not exact. An alternative approach is to linearize the equations in an $\mathrm{AC}$ feasible point. However, due to the discontinuities introduced in the problem in case of topological switching actions and contingencies, the computational burden increases considerably [55].

Another important aspect impacting the computational complexity is related to the number of binary variables in the optimization formulation 17 The binary variables introduce an exponential computational complexity in the worst case [56]. Assume that $K$ equals the number of binary variables to consider in N-0 system operation, where $K$ typically increases with the system size. The total number of binary variables in the SCOPF formulation of a reliability criterion depends on the number of system states considered in the criterion, i.e., $K \times$ the number of system states considered in the reliability criterion. Depending on the implemented risk and reliability constraints, the number of binary variables can further increase in probabilistic reliability management approaches compared to the N-1 criterion [54]. Whereas the worst-case increase of the computation time scales exponentially with the total number of binary variables, the real computation time depends on a number of factors, such as the applied solver, the optimization formulation and the problem itself. Calculation times in the range of $1.5 \mathrm{~h}$ and 9h per snapshot have been reported for a DC SCOPF implementation of resp. the N-1 criterion and a probabilistic reliability criterion applied to a real-life 450-node system [54].

The development of methods to efficiently solve large-scale, non-convex, mixed integer SCOPF formulations is subject to further research [54] ${ }^{18}$ An alternative approach to reduce the computation

\footnotetext{
${ }^{17}$ Integer variables can be approximated by their convex hull, but this results in an exponentially increasing number of additional constraints [56].

${ }^{18}$ In this discussion, we have mainly focussed on the computational challenges resulting from probabilistic reliability criteria. For a more in depth discussion of the challenges of using optimization in security analysis, we refer to [57].
} 
time uses approximations for the decision-making behaviour in time horizons closer to real-time. These approximations can be learned using supervised learning techniques and can be applied as constraints in the SCOPF formulation [58].

\subsection{Assumptions in steady-state SCOPF formulations}

To limit the computational complexity, the modeling approach of the case study incorporates a number of assumptions TSOs might be concerned about in a practical analysis. These assumptions might impact reliability management decisions, but do so for both deterministic and probabilistic methods. Moreover, the objective of this study is not to formulate the optimal reliability criterion to be used in a practical TSO context or to develop a SCOPF formulation that can be used in on-line system operation. By proposing a classification of reliability criteria based on uniform mathematical formulations, the study intends to clarify the characteristics of different reliability criteria.

First, in addition to the two decision stages considered in this paper (i.e. day-ahead operational planning and real-time operation), TSO's reliability management consists of several additional stages, e.g. the D-2 decision stage, the intraday market and the short-term preventive stage. All these decision stages could be considered in stochastic programming [17, 59, 60. Each of these decision stages is also influenced by external factors that are out of the control of the TSO, such as markets, balance responsible parties, generators and loads 19

Second, modeling all aspects of power system reliability and stability in the decision-making process is a challenging task SCOPF formulations typically use simplified steady-state formulations. First, the applied convex DC SCOPF is an approximation of the exact AC implementation. Second, the steadystate formulations do not consider transient stability issues. Stability issues due to topological actions are an important concern of system operators and should be integrated in SCOPF formulations to support TSO's decision making [61]. Topological actions can conceptually be integrated in steadystate SCOPF formulations, but the (numerical) stability of the different cases is not verified. System operators take these aspects indirectly into account, by harvesting the operators knowledge in SCOPF algorithms. They only focus on a predefined list of topological actions for which the stability is verified. Alternatively, the stability and security of a reduced set of topological actions can be verified by ex-ante dynamic simulations. The results of these dynamic simulations combined with machine

\footnotetext{
${ }^{19}$ In turn, market actors can also be affected by changing reliability management practices. For example, if the amount of preventive actions decreases, generators receive less revenue from preventive redispatch after day-ahead clearing (in single-price zones) or from security-constrained day-ahead clearing (in multi-price zones or nodal pricing).
} 
learning techniques can result in security rules that constrain system variables. These constraints can additionally be considered in the SCOPF formulation [62. Dynamic simulations are also required to determine the timing and sequence of multiple remedial actions [63.

Third, the effect of short-term probabilistic reliability management on the mid and long term also needs to be studied [6]. For example, more stressed systems could need higher maintenance expenditures and have higher losses. A more efficient use of current transmission capacity also leads to fewer additional investments.

\subsection{From steady-state SCOPF formulations to decision making in the control room}

Probabilistic reliability criteria based on SCOPF formulations cannot be applied in the control room yet due to the high computational complexity, the lack of detailed models and the lack of available and appropriate trade-off between the different performance aspects.

\subsection{Handling the trade-off between performance aspects}

The previous section's analysis made clear that moving away from the deterministic N-1 reliability criterion has a mixed impact on the different performance aspects. By considering an adequate set of 
system states and making a trade-off between preventive and corrective actions (i.e. moving from (a) to (c)), efficiency improves with little to no adverse effect on equality and service reliability. Further efficiency improvements, however, like considering load curtailment, come at the cost of reduced reliability and may have a negative impact on equality, depending on the level of system stress. Because of this trade-off between efficiency, service reliability and equality, no reliability criterion is the optimal one for every single power system stakeholder. In addition, TSOs are concerned about their reputation in terms of service reliability and end-consumers are not used to low reliability levels and large differences between consumer in terms of reliability.

To obtain an adequate reliability management approach, a transparent dialogue between power system stakeholders is important to clearly state TSOs' and society's preferences in terms of the performance trilemma between efficiency, service reliability and equality. This defines social acceptability in each of the three performance dimensions and determines how far one can go in realizing efficiency improvements at the cost of inequality and unreliability. The flexibility in making this trade-off will increase because of evolutions, such as smart technologies and flexibility devices, as well as new types of contracts enabling consumers to make reliability-based choices of electricity consumption.

Besides the quantitative performance aspects, the qualitative performance aspects should be considered in the design and selection of alternative reliability management approaches and criteria. TSOs should carry out the proposed multi-dimensional analysis for their systems to ensure practicality and applicability of the reliability management approach besides its quantitative performance ${ }^{20}$ Multiattribute utility theory can assist in the design and selection of reliability criteria. It is a statistical decision theoretic framework that facilitates the trade-off between performance aspects, both quantitative aspects with different units and qualitative aspects 68.

\section{Conclusion}

A transition towards probabilistic power system reliability criteria should be initiated to deal with the evolutions in power systems and to efficiently integrate modern technologies. However, probabilistic criteria have a steep learning curve and require additional input information, which are barriers for their practical application. The classification of criteria proposed in this paper distinguishes four characteristics that bridge the gap between the currently used deterministic N-1 criterion and an

\footnotetext{
${ }^{20}$ To ensure the applicability of probabilistic reliability management approaches in the future, the Norwegian transmission system operator Statnett has founded a data science department that focuses amongst others on the determination of detailed failure probabilities 67.
} 
unconstrained probabilistic approach that minimizes expected total system cost without considering additional constraints on service reliability. The characteristics impact the applicability, practicality and acceptability of reliability criteria, which asks for a multi-dimensional performance analysis. The proposed analysis enables a better understanding of the different reliability criteria in the literature and the difficulties for these criteria to be adequately adopted by TSOs that continue to use the N-1 criterion. The case study in this paper shows that the largest savings of expected total cost are due to a trade-off between preventive and corrective actions. A smaller portion is due to the additional inclusion of load curtailment actions in the trade-off. Limits on individual or aggregate service unreliability levels decrease service unreliability, but increase expected total costs compared to an unconstrained probabilistic approach. Inequality between consumers in terms of service reliability depends on the service reliability level and system characteristics.

It is up to TSOs to carry out the proposed multi-dimensional analysis for their systems to identify missing input information and to verify the impact of alternative reliability criteria on their own systems. This should be supported by more theoretical and applied research on the intermediate steps between the deterministic N-1 criterion and probabilistic criteria. Multi-attribute utility theory can assist in making the trade-off between the multiple quantitative and qualitative performance aspects in the design and selection of intermediate reliability criteria. Regulators should provide adequate regulation to enable the practical use of reliability criteria considering TSO's capabilities and society's preferences.

\section{Acknowledgments}

The work of Evelyn Heylen is supported by the Research Foundation Flanders (FWO). The research leading to these results has received funding from the European Union Seventh Framework Programme under Grant Agreement No 608540.

\section{References}

[1] G. Strbac, D. Kirschen, R. Moreno, Reliability standards for the operation and planning of future electricity networks, Foundations and Trends in Electric Energy Systems 1 (3) (2016) 143-219.

[2] J. McCalley, et al., Probabilistic security assessment for power system operations, IEEE Power and Energy Society General Meeting (2004). 
[3] M. Ni, J. McCalley, V. Vittal, T. Tayyib, Online risk-based security assessment, IEEE Transactions on Power Systems 18 (1) (2003) 258-265.

[4] G. Strbac, S. Ahmed, D. Kirschen, R. Allan, A method for computing the value of corrective security, IEEE Tran. Power Syst. 13 (3) (1998) 1096-1102.

[13] Y. Dai, J. McCalley, N. Abi-Samra, V. Vittal, Annual risk assessment for overload security, IEEE Trans. Power Syst. 16 (4) (2001) 616-623.

[14] H. Wan, J. McCalley, V. Vittal, Risk based voltage security assessment, IEEE Trans. Power Syst. 15 (4) (2000) 1247-1254. 
[15] S. Lee, S. Hoffman, Power delivery reliability initiative bears fruit, Computer Applications in Power, IEEE 14 (3) (2001) 56-63.

[16] M. Zima, G. Andersson, On security criteria in power systems operation, in: Power and Energy Society General Meeting, IEEE, 2005, pp. 3089-3093.

[17] E. Karangelos, L. Wehenkel, Probabilistic reliability management approach and criteria for power system real-time operation, Power Systems Computation Conference (PSCC) (2016).

[18] J. He, L. Cheng, D. S. Kirschen, Y. Sun, Optimising the balance between security and economy on a probabilistic basis, IET generation, transmission \& distribution 4 (12) (2010) 1275-1287.

[19] W. Fu, J. D. McCalley, Risk based optimal power flow, in: Power Tech Proceedings, IEEE, 2001.

[20] F. Capitanescu, M. Glavic, D. Ernst, L. Wehenkel, Contingency filtering techniques for preventive security-constrained optimal power flow, IEEE Tran. Power Syst. 22 (4) (2007) 1690-1697.

[21] F. Capitanescu, S. Fliscounakis, P. Panciatici, L. Wehenkel, Day-ahead security assessment under uncertainty relying on the combination of preventive and corrective controls to face worst-case scenarios, PSCC (2011).

[22] F. Capitanescu, S. Fliscounakis, P. Panciatici, L. Wehenkel, Cautious operation planning under uncertainties, IEEE Tran. Power Syst. 27 (4) (2012) 1859-1869.

[23] M. Vrakopoulou, S. Chatzivasileiadis, E. Iggland, M. Imhof, T. Krause, O. Mkel, J. L. Mathieu, L. Roald, R. Wiget, G. Andersson, A unified analysis of security-constrained OPF formulations considering uncertainty, risk, and controllability in single and multi-area systems, in: Bulk Power System Dynamics and Control-IX Optimization, Security and Control of the Emerging Power Grid (IREP), IEEE, 2013, pp. 1-19.

[24] F. Capitanescu, et al., State-of-the-art, challenges, and future trends in security constrained optimal power flow, Electric Power Systems Research 81 (8) (2011) 1731-1741.

[25] GARPUR consortium, D1.2 Current practices, drivers and barriers for new reliability standards, [Online] http://www .garpur-project.eu/deliverables [Accessed: 28/10/2018] (2014).

[26] E. Heylen, G. Deconinck, D. Van Hertem, Review and classification of reliability indicators for power systems with a high share of renewable energy sources, Sustainable and Renwable Energy Reviews 97 (2018) $554-568$. 
[27] G. Strbac, P. Djapic, External peer review of grid reliability standards in new zealand: Summary report, Tech. rep., Imperial College London (2008).

[28] Task Force C4.601 CIGRE, Review of the current status of tools/techniques for risk based and probabilistic planning in power systems, Tech. rep., CIGRE (2010).

[29] GARPUR consortium, D2.2 guidelines for implementing the new reliability assessment and optimization methodology, [Online] http://www.garpur-project.eu/deliverables [Accessed: 28/10/2018] (2016).

[30] International Electrotechnical Commission and others, Electropedia: The world's online electrotechnical vocabulary, [Online] http://www.electropedia.org/ [Accessed: 28/10/2018] (2016).

[31] E. Heylen, W. Labeeuw, G. Deconinck, D. Van Hertem, Framework for evaluating and comparing performance of power system reliability criteria, IEEE Tran. Power Syst. 31 (3) (2016) 5153-5162.

[32] F. Capitanescu, et al., State-of-the-art, challenges, and future trends in security constrained optimal power flow, Electric Power Systems Research 81 (8) (2011) 1731-1741.

[33] F. M. Baldursson, E. Lazarczyk, M. Ovaere, S. Proost, Cross-Border Exchange and Sharing of Generation Reserve Capacity, The Energy Journal 39 (4) (2018) 57-83.

[34] D. Bertsimas, D. B. Brown, C. Caramanis, Theory and applications of robust optimization, SIAM review 53 (3) (2011) 464-501.

[35] D. Kirschen, G. Strbac, Why investments do not prevent blackouts, The Electricity Journal 17 (2) (2004) 29-36.

[36] National Grid, National electricity transmission system security and quality of supply

घ standard, [Online] https://www.nationalgrid.com/sites/default/files/documents/NETS\% 20SQSS\%20V2.3.pdf [Accessed: 28/10/2018] (2017).

[37] R. Scott, P. E. Dahman, N-1-1 Contingency Analysis using PowerWorld Simulator, [Online] https://www.powerworld.com/files/SimulatorN-1-1.pdf [Accessed: 28/10/2018] (October 2012). 
[38] Standard TPL-001-1 System Performance Under Normal Conditions, [Online] https://www.aeso.ca/rules-standards-and-tariff/alberta-reliability-standards/ tpl-001-system-performance-under-normal-conditions/ [Accessed: 28/10/2018] (April 2005).

[39] R. L.-Y. Chen, N. Fan, J.-P. Watson, N-1-1 contingency-constrained grid operations, in: FERC Technical Conference, 2013.

[40] A. Monticelli, M. V. F. Pereira, S. Granville, Security-constrained optimal power flow with postcontingency corrective rescheduling, IEEE Trans. Power Syst. 2 (1) (1987) 175-180.

[41] J. Condren, T. W. Gedra, P. Damrongkulkamjorn, Optimal power flow with expected security costs, IEEE Trans. Power Syst. 21 (2) (2006) 541-547.

[42] M. Ovaere, E. Heylen, S. Proost, G. Deconinck, D. Van Hertem, How detailed value of lost load data impact power system reliability decisions: a trade-off between efficiency and equality, KU Leuven Department of Economics Discussion Paper Series 16.26.

[43] F. Capitanescu, Enhanced risk-based scopf formulation balancing operation cost and expected voluntary load shedding, Electric Power Systems Research 128 (2015) 151-155.

[44] E. Heylen, M. Ovaere, S. Proost, G. Deconinck, D. Van Hertem, Fairness and Inequality in Power System Reliability: Summarizing Indices, Electric Power Systems Research (Submitted).

[45] R. Billinton, et al., A reliability test system for educational purposes-basic data, IEEE Tran. on Power Syst. 4 (3) (1989) 1238-1244.

[46] C. Grigg, et al., The IEEE reliability test system-1996. A report prepared by the reliability test system task force of the application of probability methods subcommittee, IEEE Tran. Power Syst. 14 (3) (1999) 1010-1020.

[47] E. Heylen, Evaluation of power system reliability management: Towards socially acceptable shortterm reliability criteria, Ph.D. thesis, KU Leuven (2018).

[48] EnergiNorge, Samfunnsøkonomiske Kostnader Ved Avbrudd Og Spenningsforstyrrelser, no. 349, 2012.

[49] R. Fourer, D. Gay, B. Kernighan, A modeling language for mathematical programming, Management Science 36 (1990) 519-554. 
[50] T. Van Acker, D. Van Hertem, Linear representation of preventive and corrective actions in OPF models, in: Young researchers symposium, IEEE IAS/PELS/PES Benelux Chapter, 2016.

[51] F. P. Coolen, M. C. Troffaes, T. Augustin, Imprecise probability, in: International encyclopedia of statistical science, Springer, 2011, pp. 645-648.

[52] L. Platbrood, H. Crisciu, F. Capitanescu, L. Wehenkel, Solving very large-scale securityconstrained optimal power flow problems by combining iterative contingency selection and network compression, PSCC (2011).

[53] S. Boyd, L. Vandenberghe, Convex optimization, 7th Ed., Cambridge university press, New York, 2004.

[54] GARPUR consortium, D7.3 Report on a broader comparison of different reliability criteria including recommendations on how to evolve into an industry grade tool, [Online] http://www. garpur-project.eu/deliverables [Accessed: 28/10/2018] (2017).

[55] B. Stott, J. Jardim, O. Alsa, Dc power flow revisited, IEEE Tran. Power Syst. 24 (3) (2009) $1290-1300$.

[56] H. P. Williams, Logic and Integer programming, 1st Ed., Springer, New York, 2009.

[57] B. Stott, O. Alsac, A. J. Monticelli, Security analysis and optimization, Proceedings of the IEEE 75 (12) (1987) 1623-1644.

[58] L. Duchesne, E. Karangelos, L. Wehenkel, Machine learning of real-time power systems reliability management response, in: PowerTech, IEEE, 2017.

[59] V. V. Vadlamudi, C. Hamon, G. Kjølle, S. Perkin, On improving data and models on corrective control failures for use in probabilistic reliability management, International Conference on Probabilistic Methods Applied to Power Systems (PMAPS) (2016) 1-6.

[60] J. L. Calvo, S. H. Tindemans, G. Strbac, Incorporating failures of system protection schemes into power system operation, Sustainable Energy, Grids and Networks 8 (2016) 98-110.

645

[61] GARPUR consortium, D8.2 Results from pilot testing using the quantification platform prototype, [Online] http://www . garpur-project.eu/deliverables [Accessed: 28/10/2018] (2017). 
[62] J. L. Cremer, I. Konstantelos, S. H. Tindemans, G. Strbac, Data-driven Power System Operation: Exploring the Balance between Cost and Risk, IEEE Transactions on Power Systems (2018) - .

[63] F. Capitanescu, T. Van Cutsem, L. Wehenkel, Coupling optimization and dynamic simulation for preventive-corrective control of voltage instability, IEEE Transactions on Power Systems 24 (2) (2009) 796-805.

[64] GARPUR consortium, D6.2 How to upgrade reliability management for short-term decision making, [Online] http://www .garpur-project.eu/deliverables [Accessed: 28/10/2018] (2016).

[65] GARPUR consortium, D8.3 Results from near real life pilot testing (Public Summary), [Online] http://www.garpur-project.eu/deliverables [Accessed: 28/10/2018] (2017).

[66] S. Perkin, Real-time Weather-dependent Probabilistic Reliability Assessment of the Icelandic Power System, Ph.D. thesis, Reykjavik University (2018).

[67] T. Trötscher, Estimating the probability of failure for over-

1. head lines, [Online]: https://datascience.statnett.no/2018/04/23/ estimating-probability-of-failure-overhead-line-lightning/ [Accessed: 28/10/2018] (April 2018).

[68] R. L. Keeney, Decisions with multiple objectives : preferences and value tradeoffs, Wiley, London, 1976. 